\title{
Metabolism of Strained Rings: Glutathione S-transferase-Catalyzed Formation of a Glutathione-Conjugated Spiro-azetidine without Prior Bioactivation
}

\author{
Xue-Qing Li, Gunnar Grönberg, Eva-Henriette Bangur, Martin A. Hayes, \\ Neal Castagnoli, Jr., and (1)Lars Weidolf
}

Drug Metabolism and Pharmacokinetics, Research and Early Development Cardiovascular, Renal and Metabolism (X.-Q.L., E.-H.B., L.W.), Hit Discovery, Discovery Sciences (M.A.H.), and Medicinal Chemistry, Early Respiratory, Inflammation and Autoimmunity (G.G.), BioPharmaceuticals R\&D, AstraZeneca, Gothenburg, Sweden; and Department of Chemistry, Virginia Tech, Blacksburg, Virginia (N.C.J.)

Received July 8, 2019; accepted August 28, 2019

\section{ABSTRACT}

AZD1979 [(3-(4-(2-oxa-6-azaspiro[3.3]heptan-6-ylmethyl)phenoxy) azetidin-1-yl)(5-(4-methoxyphenyl)-1,3,4-oxadiazol-2-yl)methanone] is a melanin-concentrating hormone receptor 1 antagonist designed for the treatment of obesity. In this study, metabolite profiles of AZD1979 in human hepatocytes revealed a series of glutathionerelated metabolites, including the glutathionyl, cysteinyl, cysteinylglycinyl, and mercapturic acid conjugates. The formation of these metabolites was not inhibited by coincubation with the cytochrome P450 (P450) inhibitor 1-aminobenzotriazole. In efforts to identify the mechanistic features of this pathway, investigations were performed to characterize the structure of the glutathionyl conjugate M12 of AZD1979 and to identify the enzyme system catalyzing its formation. Studies with various human liver subcellular fractions established that the formation of M12 was $N A D(P) H$-independent and proceeded in cytosol and $\mathrm{S} 9$ fractions but not in microsomal or mitochondrial fractions. The formation of M12 was inhibited by ethacrynic acid, an inhibitor of glutathione S-transferases (GSTs). Several human recombinant GSTs, including GSTA1, A2-2, M1a, M22, T1-1, and GST from human placenta, were incubated with AZD1979. All GSTs tested catalyzed the formation of M12, with
GSTA2-2 being the most efficient. Metabolite M12 was purified from rat liver S9 incubations and its structure elucidated by NMR. These results establish that M12 is the product of the GST-catalyzed glutathione attack on the carbon atom $\alpha$ to the nitrogen atom of the strained spiro-azetidinyl moiety to give, after ring opening, the corresponding amino-thioether conjugate product, a direct conjugation pathway that occurs without the prior substrate bioactivation by P450.

\section{SIGNIFICANCE STATEMENT}

The investigated compound, AZD1979, contains a 6-substituted-2oxa-6-azaspiro[3.3]heptanyl derivative that is an example of strained heterocycles, including spiro-fused ring systems, that are widely used in synthetic organic chemistry. An unusual azetidinyl ringopening reaction involving a nucleophilic attack by glutathione, which does not involve prior cytochrome P450-catalyzed bioactivation of the substrate and which is catalyzed by glutathione transferases, is reported. We propose a mechanism involving the protonated cyclic aminyl intermediate that undergoes nucleophilic attack by glutathione thiolate anion in this reaction, catalyzed by glutathione transferases.

\section{Introduction}

AZD1979 [(3-(4-(2-oxa-6-azaspiro[3.3]heptan-6-ylmethyl)phenoxy) azetidin-1-yl)(5-(4-methoxyphenyl)-1,3,4-oxadiazol-2-yl)methanone] is a melanin-concentrating hormone receptor 1 antagonist designed for the treatment of obesity by utilizing its appetite-modulating properties (Johansson et al., 2016). AZD1979 (Fig. 1) contains a spirooxetanylazetidinyl moiety that confers favorable physicochemical and pharmacokinetic properties in comparison with other analogs with simple 4-, 5-, or 6-membered ring systems (Johansson et al., 2016). Previous studies from this laboratory revealed a novel metabolic

The authors declare that there are no potential conflicts of interest. https://doi.org/10.1124/dmd.119.088658. pathway on the spiro-ring system: the microsomal epoxide hydrolase catalyzed hydrolysis of the spiro-oxetanyl ring of AZD1979, forming the corresponding ring-opened diol product ( $\mathrm{Li}$ et al., 2016). In the present study, we investigated an additional unusual non-cytochrome P450 (nonP450)-dependent metabolic pathway: glutathione (GSH) conjugation of the parent with the modification located again on the spiro-ring system.

Electrophilic species, whether inherent in a drug molecule or formed via metabolic activation, is of toxicological concern in drug design and development. GSH conjugation is an important detoxification pathway of such electrophilic species and has been studied extensively (Munter et al., 2003; Dragovic et al., 2010), and consequently, drug candidates that form GSH adducts often are redesigned to avoid this liability (Okada et al., 2011; Zhuo et al., 2015; Dekker et al., 2016). The principal pathway for the formation of GSH conjugates of xenobiotics involves

ABBREVIATIONS: 1-ABT, 1-aminobenzotriazole; GSH, glutathione; GST, glutathione S-transferase; GST-PLA, GST from human placenta; HMBC, heteronuclear multiple-bond correlation; LC, liquid chromatography; MS, mass spectrometry; MS/MS, tandem mass spectrometry; P450, cytochrome P450; ROESY, rotating frame nuclear Overhauser effect spectroscopy; SPE, solid-phase extraction; UPLC-HRMS, ultrahighperformance liquid chromatography-high-resolution mass spectrometry. 
<smiles>COc1ccc(-c2nnc(C(=O)N3CC(Oc4ccc(CN5CC6(COC6)C5)cc4)C3)o2)cc1</smiles>

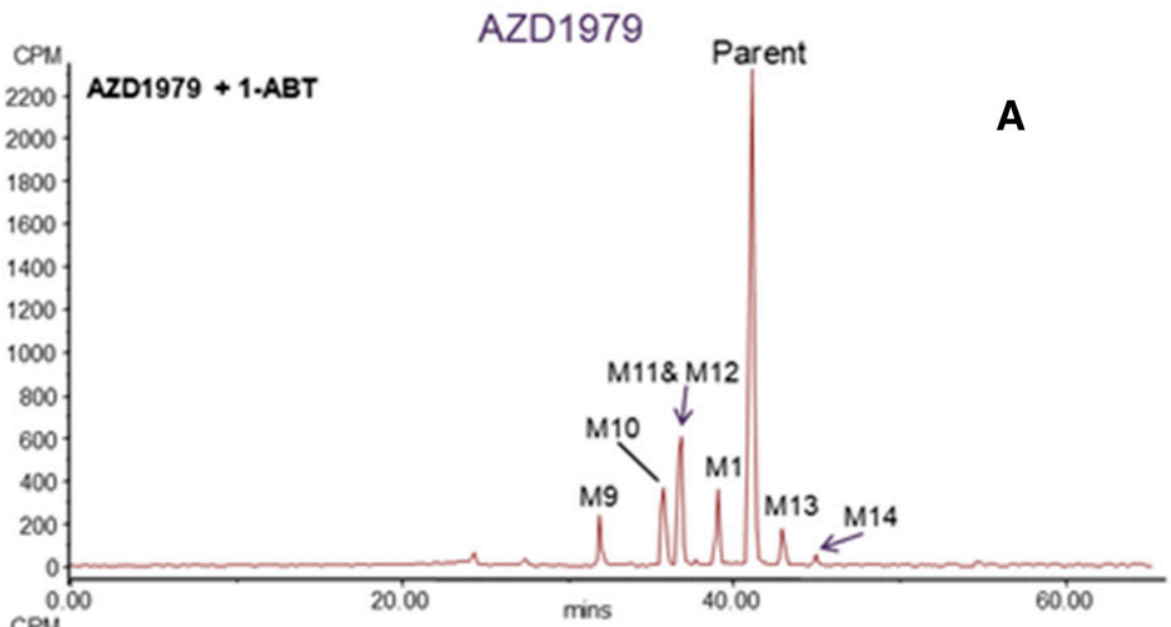

B

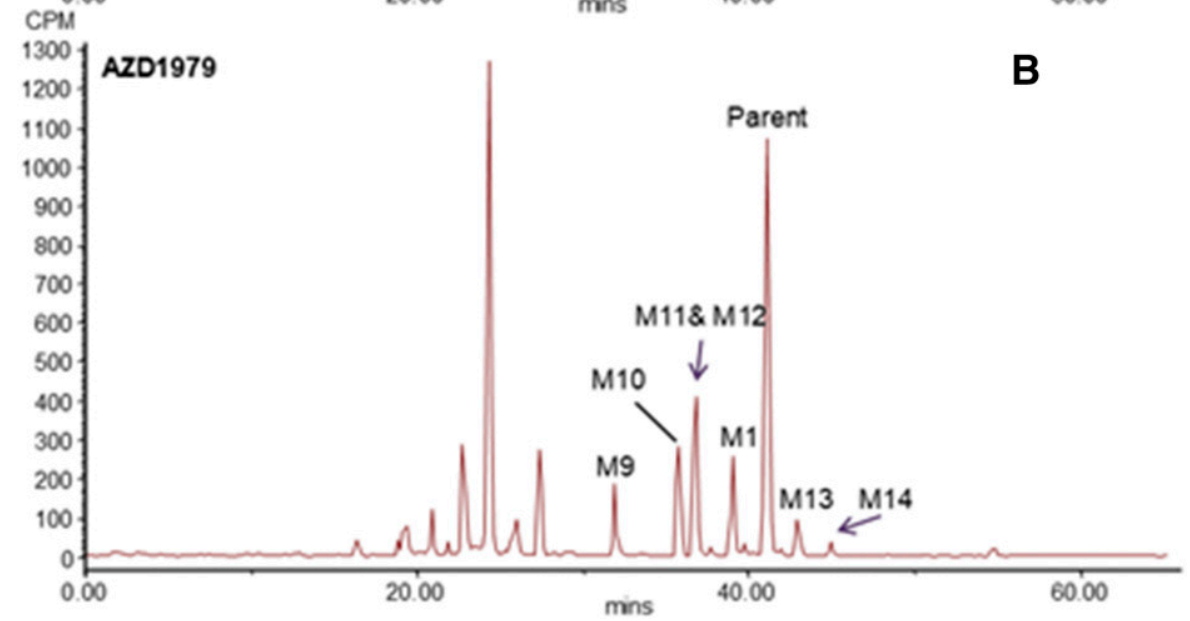

Fig. 1. Radiochromatograms of $\left[{ }^{14} \mathrm{C}\right] \mathrm{AZD} 1979$ and its metabolites formed in human hepatocytes in the presence (A) and absence (B) of 1-ABT following 3 hours of incubation. Asterisk indicates the ${ }^{14} \mathrm{C}$-labeled position. an initial two-electron oxidation generally catalyzed by a member of the P450 family of enzymes, generating a reactive electrophilic intermediate, e.g., an arene oxide, a quinone/iminoquinone/quinone methide, or a carbinol, such as a hydroxy ketone that can undergo spontaneous dehydration to form a reactive Michael acceptor (e.g., an $\alpha, \beta$-enone). In this study, we sought to examine the pathway leading to this GSH conjugate to understand its potential contribution to the safety profile of AZD1979. We report the structural characterization of the conjugate and the dependence of conjugate formation on various glutathione $S$-transferase (GST) enzyme systems, but not on prior $\mathrm{P} 450$-mediated activation of the parent molecule. Finally, we propose a mechanism to account for the formation of this metabolite. AZD1979 was incubated initially in human hepatocytes and later in various human liver subcellular fractions, in the presence or absence of NADH/NADPH and GSH, and in the presence of chemical inhibitors of the enzymes under study. Experiments were also performed to understand the catalytic activities of recombinant or isolated GSTs against AZD1979 and its chemical reactivity with GSH in aqueous buffer. In addition, sufficient quantities of the GSH conjugate M12 were purified from scale-up incubations in rat liver S9 fraction to allow the unambiguous structural elucidation of M12 by NMR. In summary, we report a non-P450-mediated GSH conjugation of AZD1979 with focus on the enzymes involved in this metabolic reaction. We include a full structural characterization of M12 and propose a mechanism for its formation.

\section{Materials and Methods}

Chemicals and Reagents. AZD1979, 1-aminobenzotriazole (1-ABT), cysteine, $\mathrm{N}$-acetylcysteine, and ethacrynic acid were obtained from AstraZeneca Compound Management, AstraZeneca (Gothenburg, Sweden). $\left[{ }^{14} \mathrm{C}\right]$-Labeled AZD1979 (specific radioactivity $2.1 \mathrm{MBq} / \mathrm{nmol}$, radiochemical purity $99 \%$ ) was obtained from Isotope Chemistry, AstraZeneca. L-Glutathione in reduced form, NADPH, NADH, GST from human placenta (GST-PLA), and Williams' E medium without phenol red were purchased from Sigma-Aldrich Chemie $\mathrm{GmbH}$ (Schnelldorf, Germany). Cryopreserved pooled human hepatocytes were purchased from BioIVT (Baltimore, MD). A pool of human liver microsomes was purchased from BD Gentest (Woburn, MA). Pooled human liver mitochondria, cytosol, and S9 fractions were purchased from XenoTech, LLC (Kansas City, KS). Pooled rat liver S9 fraction was obtained from Bioreclamation IVT (Frankfurt, Germany). Recombinant human GSTA2-2, GSTM2-2, and GSTT11 were purchased from Oxford Biomedical Research (Rochester Hills, MI), and GSTM1a (Lys-173) and GSTA1 were obtained from Cypex (Dundee, Scotland, UK). All other chemicals and solvents were of the highest quality commercially available.

Incubations in Human Hepatocytes. $\left[{ }^{14} \mathrm{C}\right] \mathrm{AZD} 1979$ was incubated with human hepatocyte suspensions in Williams' $\mathrm{E}$ buffer ( $\mathrm{pH}$ 7.4) in the absence and presence of 1-ABT, a nonspecific P450 inhibitor (Emoto et al., 2003). Each 
incubation $(200 \mu \mathrm{l})$ contained 2 million cells/ml, $\left[{ }^{14} \mathrm{C}\right] \mathrm{AZD} 1979(10 \mu \mathrm{M})$, with or without 1-ABT $(1 \mathrm{mM})$, in a flat-bottomed glass vial at $37^{\circ} \mathrm{C}, 95 \%$ air, and $5 \% \mathrm{CO}_{2}$ at saturated humidity. After a 10 -minute preincubation at $37^{\circ} \mathrm{C},\left[{ }^{14} \mathrm{C}\right]$ AZD1979 was added to start the reaction. At 0 and 3 hours, the reactions were stopped by adding $400 \mu \mathrm{l}$ of ice-cold acetonitrile and kept on ice for at least 30 minutes before centrifugation at $10,000 \mathrm{~g}$ and $4^{\circ} \mathrm{C}$ for 10 minutes. The supernatants were diluted with one volume of water and analyzed by ultrahighperformance liquid chromatography-high-resolution mass spectrometry (UPLCHRMS) and radiochemical detection to record metabolite profiles.

Formation of the GSH-Conjugated Metabolite M12 in Human Liver Subcellular Fractions. Incubation experiments with human liver fractions, i.e., microsomes, cytosol, $\mathrm{S} 9$, or mitochondria, were performed at a protein concentration of $1 \mathrm{mg} / \mathrm{ml}$ in $0.1 \mathrm{M}$ potassium phosphate buffer ( $\mathrm{pH}$ 7.4) with GSH $(2 \mathrm{mM})$ and $\mathrm{MgCl}_{2}(3 \mathrm{mM})$ with or without $\mathrm{NAD}(\mathrm{P}) \mathrm{H}$ (mixture of NADPH and $\mathrm{NADH}, 1 \mathrm{mM}$ each) at an AZD1979 concentration of $10 \mu \mathrm{M}$. The incubations were performed in a 96 -well format with a total volume of $100 \mu \mathrm{l} /$ well at $37^{\circ} \mathrm{C}$ in a shaking incubator. After 5-minute preincubation, the reaction was initiated by addition of AZD1979. Following a defined incubation time of 0 and 60 minutes, ice-cold acetonitrile $(200 \mu \mathrm{l})$ was added to the reaction mixture to stop the reaction. After centrifugation at $4500 \mathrm{~g}$ for 20 minutes, a $50-\mu \mathrm{l}$ aliquot of the reaction supernatant was diluted with $100 \mu \mathrm{l}$ of water before UPLC-HRMS analysis.

The inhibitory effects of ethacrynic acid, a GST inhibitor (Ploemen et al., 1990), on the formation of M12 were examined. AZD1979 $(20 \mu \mathrm{M})$ was incubated with and without the addition of $100 \mu \mathrm{M}$ ethacrynic acid in human liver S9 fractions at a protein concentration of $1 \mathrm{mg} / \mathrm{ml}$ in $0.1 \mathrm{M}$ potassium phosphate buffer ( $\mathrm{pH}$ 7.4) containing $2 \mathrm{mM}$ GSH for 0 and 60 minutes.

In the $\mathrm{pH}$ dependency study, AZD1979 $(20 \mu \mathrm{M})$ was incubated for 0 and 60 minutes in human liver S9 fractions ( $1 \mathrm{mg} / \mathrm{ml}$ protein) containing $2 \mathrm{mM} \mathrm{GSH}$, in $0.1 \mathrm{M}$ potassium phosphate buffer at $\mathrm{pH} 6.5,7.4,8.5$, and 9.0. At the end of incubation, samples were processed as described earlier by protein precipitation, and the formation of M12 was analyzed by UPLC-HRMS.

In Vitro Incubation of AZD1979 with Human GSTs. AZD1979 was incubated with recombinant human GSTs and GST-PLA and supplemented with GSH. The incubation mixture contained $10 \mu \mathrm{M}$ AZD1979, $2 \mathrm{mM}$ GSH, and a single GST in $0.1 \mathrm{M}$ potassium phosphate buffer $(\mathrm{pH} 6.5)$ at $37^{\circ} \mathrm{C}$ for 0,60 , and 120 minutes. GSTs were purchased from different commercial sources, so the protein concentrations used in the incubations were altered accordingly, with 0.1 and $0.5 \mathrm{mg} / \mathrm{ml}$ for GSTM1a, GSTA-1, and GST-PLA, and at $0.5 \mathrm{U} / \mathrm{ml}$ for GSTA2-2 (0.03 mg/ml), GSTM2-2 (0.005 mg/ml), and GSTT1-1 $(0.02 \mathrm{mg} / \mathrm{ml})$, respectively. Samples were processed as described earlier by protein precipitation, and the formation of M12 was monitored by UPLC-HRMS.

Chemical Reactivity of AZD1979. Incubations to examine the chemical reactivity of AZD1979 $(50 \mu \mathrm{M})$ were performed in the presence of GSH $(5 \mathrm{mM})$, $\mathrm{N}$-acetylcysteine $(5 \mathrm{mM})$, or cysteine $(1 \mathrm{mM})$ in $0.1 \mathrm{M}$ phosphate buffer at $\mathrm{pH} 6.5$, 7.4 , and 9.0, respectively, at 37 and $60^{\circ} \mathrm{C}$. The reactions were stopped at $0,1,2$, and 3 hours by adding an equal volume of ice-cold acetonitrile and then analyzed immediately by UPLC-HRMS.

Biosynthesis of GSH-Conjugated Metabolite M12. Initial investigations showed that rat liver $\mathrm{S} 9$ fractions catalyzed the formation of the same GSH adduct of AZD1979 as in human hepatocytes by UPLC-HRMS analysis (data not shown). Therefore, rat liver S9 was used as the biocatalyst for the synthesis of M12. The reaction contained $300 \mu \mathrm{M}$ AZD1979, $3 \mathrm{mg} / \mathrm{ml}$ rat liver S9 fractions, $0.1 \mathrm{M}$ phosphate buffer ( $\mathrm{pH} 7.4)$, and $5 \mathrm{mM} \mathrm{GSH}$. The reaction mixture $(200 \mathrm{ml})$ was incubated at $37^{\circ} \mathrm{C}$ for 20 hours in a conical flask $(500 \mathrm{ml})$. The flask was shaken on an orbital shaker at $200 \mathrm{rpm}$, and the reaction was monitored by UPLCHRMS. After 20-hour incubation, the reaction mixture was quenched using icecold acetonitrile:methanol $[1: 1(\mathrm{v} / \mathrm{v}) ; 600 \mathrm{ml}]$, and the solution was allowed to stand in a refrigerator at $4^{\circ} \mathrm{C}$ for 60 minutes. Following centrifugation for 20 minutes at $4000 \mathrm{~g}$ and $4^{\circ} \mathrm{C}$, the supernatant was transferred to a round-bottomed flask and concentrated on a rotary evaporator at room temperature. After removal of the organic solvents, the aqueous fraction was subjected to solid-phase extraction (SPE) using Oasis HLB SPE cartridges ( $6 \mathrm{ml}, 200 \mathrm{mg}$ sorbent; Waters, Milford, MA). The SPE cartridges were preconditioned with acetonitrile $(6 \mathrm{ml})$ followed by water $(6 \mathrm{ml})$. After loading the sample, the cartridges were washed with water $(6 \mathrm{ml})$ and eluted with acetonitrile $(2 \times 6 \mathrm{ml})$. The acetonitrile eluates were evaporated to dryness under a stream of nitrogen at room temperature. The crude residue was reconstituted in $30 \%$ acetonitrile/water and fractionated by semipreparative liquid chromatography (LC). The LC system consisted of a Shimadzu SCL-10AD system controller with a CTC HTS PAL autosampler (Presearch, Hitchin, UK), an SPD-10AD UV detector, and two LC-10AD pumps. The sample separation and purification were performed using an Xbridge prep BEH130 $\mathrm{C}_{18}$ column $(10 \times 100 \mathrm{~mm}, 5 \mu \mathrm{m}$; Waters $)$. A gradient elution was performed at a flow rate of $4.5 \mathrm{ml} / \mathrm{min}$ using aqueous $0.1 \%$ formic acid (solvent $\mathrm{A}$ ) and acetonitrile (solvent $\mathrm{B}$ ) with the following composition: 0-14 minutes, $10 \%$ B ; 14-16 minutes, $10 \%-90 \%$ B; 16-22 minutes, $90 \%$ B. The column was reequilibrated at $10 \% \mathrm{~B}$ for 8 minutes before the next injection. The collection of M12-containing fractions was based on UV detection at a wavelength of $256 \mathrm{~nm}$. Using this chromatographic system, M12 eluted at 13.6 minutes, well resolved from the polar impurity fractions and the parent compound (which eluted after 16 minutes).

The M12-containing fractions were combined, concentrated, and freeze-dried. A further SPE extraction of the M12-containing residue was performed using deuterium-labeled solvent, and the resulting eluate of M12 was then directly subjected for NMR characterization. The combined residue obtained from the semipreparative LC was dissolved in $0.5 \mathrm{ml}$ of acetonitrile- $\mathrm{D}_{3}: \mathrm{D}_{2} \mathrm{O}(1: 2)$, diluted with $10 \mathrm{ml} \mathrm{D}_{2} \mathrm{O}$, and purified once again using an Oasis HLB SPE cartridge $(1 \mathrm{ml}$, $30 \mathrm{mg}$ sorbent; Waters). The cartridge was preconditioned using acetonitrile- $\mathrm{D}_{3}$ followed by $\mathrm{D}_{2} \mathrm{O}$. The sample was loaded on the cartridge, washed with $\mathrm{D}_{2} \mathrm{O}$, dried under vacuum for 5 minutes, eluted with acetonitrile- $\mathrm{D}_{3}: \mathrm{D}_{2} \mathrm{O}(1: 1)$, and the final eluate was transferred to a 5-mm NMR tube for NMR analyses. The UPLC-HRMS method described earlier was used to confirm the identity of M12.

NMR Characterization of GSH-Conjugated Metabolite M12. NMR spectra were acquired for the isolated M12 on 600-MHz Bruker Avance I or Avance III NMR spectrometers equipped with 5-mm TCI or QNP cryo probes. 1D ${ }^{1} \mathrm{H},{ }^{13} \mathrm{C}$, and $2 \mathrm{D}$ experiments [H,H-correlation spectroscopy (COSY), H,H-rotating frame nuclear Overhauser effect spectroscopy (ROESY), C,H-heteronuclear single quantum correlation (HSQC), and C,H-(long-range)-heteronuclear multiple-bond correlation (HMBC)] were performed using standard Bruker pulse sequences at $25^{\circ} \mathrm{C}$. The NMR spectrum of the parent AZD1979 (approximately $5 \mathrm{mg}$ dissolved in methanol- $\mathrm{D}_{4}$ ) was also acquired for comparison. Chemical shifts are expressed relative to the internal residual resonance of the deuterated solvent, $\delta 3.33 / 49.0$ $\left({ }^{1} \mathrm{H} /{ }^{13} \mathrm{C}\right) \mathrm{ppm}$ for methanol- $\mathrm{D}_{4}$ and $\delta 2.36 / 0.88 \mathrm{ppm}$ for acetonitrile- $\mathrm{D}_{3}: \mathrm{D}_{2} \mathrm{O}$.

UPLC-HRMS and Radiochemical Detection Analysis. For metabolite profiling of ${ }^{14} \mathrm{C}$-labeled AZD1979 in human hepatocytes, a Waters Acquity UPLC system coupled with a Synapt G2 quadrupole time-of-flight mass spectrometer (Waters) was used. Sample separation was performed on an Acquity BEH $\mathrm{C}_{18}$ column $(3.0 \times 150 \mathrm{~mm}, 1.7 \mu \mathrm{m}$; Waters $)$ with mobile phase $\mathrm{A}$ ( $0.1 \%$ formic acid in water) and mobile phase B ( $100 \%$ acetonitrile). The flow rate was $0.8 \mathrm{ml} / \mathrm{min}$, and the column temperature was maintained at $45^{\circ} \mathrm{C}$ in a column oven. The initial mobile phase consisted of $95 \%$ A and $5 \%$ B. Solvent B was increased linearly to $18 \%$ over 45 minutes, to $25 \%$ over the next 5 minutes, and to $65 \%$ over a further 5 minutes. Solvent B was then increased to $95 \%$ and was maintained at this level for 5 minutes followed by a 5 -minute re-equilibration step. The eluent from the analytical column was split with $20 \%$ directed to the mass spectrometer and the remainder to a fraction collector (Gilson, Inc., Middleton, WI) for collection in 96-well Luma plates (PerkinElmer Life and Analytical Sciences) with a collection rate of 10 seconds/well. The plates were evaporated to dryness in a ventilated area and counted for radioactivity (4 minutes/well) using a TopCount Microplate scintillation Counter (PerkinElmer). The data were exported to Laura (version 4; LabLogic) for peak integration after background subtraction. The Synapt G2 quadrupole time-of-flight mass spectrometer was equipped with an electrospray interface and operated in positive ionization mode. Specific mass spectrometric source conditions were as follows: capillary voltage of $0.5 \mathrm{kV}$, sample cone voltage of $30 \mathrm{~V}$, and a desolvation temperature of $450^{\circ} \mathrm{C}$. Mass spectra were acquired over the range $m / z$ 80-1200. Data were acquired in full-scan mass spectrometry (MS) and tandem mass spectrometry (MS/MS) modes using leucine-enkephalin as an internal calibrant for the accurate mass. All MS data were acquired using a low collision voltage $(6 \mathrm{~V})$ and a high-voltage ramp (15-30 V). MS/MS data were acquired using a transfer collision energy of $20 \mathrm{eV}$ and trap collision energy ramped from 15 to $35 \mathrm{eV}$. MassLynx (version 4.1) was used to control the system, data acquisition, and analysis. MetaboLynx software (Waters) was used to process the data for metabolite identification. Metabolite structures were assigned based on their HRMS and MS/MS production mass spectra. 
UPLC-HRMS Analysis of M12. For the analysis of metabolite M12 in human liver subcellular fractions and GSTs, the same UPLC-HRMS system as described earlier was used but with modified LC chromatographic conditions. Sample separation was carried on an Acquity UPLC BEH C 18 column $(2.1 \times 100$ $\mathrm{mm}, 1.7 \mu \mathrm{m}$; Waters) with mobile phase $\mathrm{A}(0.1 \%$ formic acid in water) and mobile phase B (100\% acetonitrile). A linear gradient was carried out at a constant flow rate of $0.5 \mathrm{ml} / \mathrm{min}$ from $10 \% \mathrm{~B}$ to $40 \% \mathrm{~B}$ over $0-6$ minutes followed by 1 -minute re-equilibration step. The injection volume was $5 \mu \mathrm{l}$, and the column temperature was maintained at $45^{\circ} \mathrm{C}$ in a column oven. Metabolite structures were assigned based on their HRMS and MS/MS product-ion mass spectra. TargetLynx was used for quantitative analysis by integration of the peak areas of the compound of interest from extracted ion chromatograms using a mass window of $20 \mathrm{mDa}$ centered on the theoretical $\mathrm{m} / \mathrm{z}$ of M12 (doubly and singly charged ions at $m / z, 385.6446+770.281)$ and AZD1979 $(\mathrm{m} / \mathrm{z}$ 463.1976) for comparison. The means of duplicate analyses were used for data analysis.

\section{Results}

Metabolite Profiles of $\left[{ }^{14} \mathrm{C}\right] A Z D 1979$ in Human Hepatocyte Incubations in the Presence and Absence of 1-ABT. Radiochromatograms (Fig. 1) showed that a number of metabolites were formed by $\left[{ }^{14} \mathrm{C}\right]$ AZD1979 in human hepatocyte incubations. Among these metabolites, the formation of M1 and M9-M14 was not affected significantly in the presence of the nonselective P450 inhibitor 1-ABT (Emoto et al., 2003). The HRMS spectrum (Fig. 2A) of metabolite M12 (the non$\mathrm{P} 450$-mediated formation) showed that its accurate mass $\left([\mathrm{M}+\mathrm{H}]^{+} \mathrm{m} / \mathrm{z}\right.$ 772.2714) was $307.0706 \mathrm{Da}$ higher than that of the parent (theoretical $[\mathrm{M}+\mathrm{H}]^{+} \mathrm{m} / \mathrm{z}$ 465.2008*, where $*$ indicates the ${ }^{14} \mathrm{C}$-isotopolog). The observed mass defect suggested an elemental composition of $\mathrm{C}_{10} \mathrm{H}_{17} \mathrm{~N}_{3} \mathrm{O}_{6} \mathrm{~S}$ consistent with a molecule of glutathione attached to the parent compound. Furthermore, the MS/MS product ions of M12 (Fig. 2A) showed a fragment ion at $\mathrm{m} / \mathrm{z}, 643.2986$ corresponding to the typical neutral loss of pyroglutamic acid (129 Da from the glutathionyl moiety), confirming that M12 is a glutathionyl conjugate of AZD1979. Additional metabolites that, according to accurate mass data, are most likely derived from M12 include M9 (cysteinylglycinyl metabolite, $[\mathrm{M}+\mathrm{H}]^{+} \mathrm{m} / \mathrm{z}, 641.2308^{*}$ ), M10 (cysteinyl metabolite, $[\mathrm{M}+\mathrm{H}]^{+} \mathrm{m} / \mathrm{z}$ $586.2195^{*}$ ), and M14 (mercapturic acid conjugate, $[\mathrm{M}+\mathrm{H}]^{+} \mathrm{m} / \mathrm{z}$ $\left.628.2294^{*}\right)$. Figure 3 illustrates the proposed glutathione conjugation metabolic pathways for AZD1979. All four glutathione-related metabolites accounted for approximately $10 \%$ of the total radioactivity (constituting $16 \%$ of the sum of radioactivity derived from all metabolites) in the sample following a 3-hour incubation, indicating that glutathione conjugation and subsequent formation of GSH-related breakdown products play an important role in the metabolism of AZD1979. The remaining three metabolites, the formation of which was not inhibited by $1-\mathrm{ABT}$, were subsequently characterized as an $N$-glucuronide (M11) and an $N$-oxide (M13) of AZD1979, with the biotransformation occurring on the spiro-ring system (data not shown). Full characterization of the third metabolite revealed its formation via microsomal epoxide hydrolase to the oxetane ring-opened diol (M1) (Li et al., 2016).

Analysis of MS/MS spectra (Fig. 2A) showed that the major fragment ions of M12 were derived from the neutral losses containing the spirooxetanylazetidinyl moiety, giving identical fragment ions to those observed in the parent molecule $(\mathrm{m} / \mathrm{z}$ 366.1224*, 260.0486*, and $\left.177.0419^{*}\right)$. The accurate mass assignments of the fragment ions are shown in Fig. 2. These assignments are also supported by the MS/MS spectra of unlabeled M12 (Fig. 2B). We conclude that addition of GSH had occurred with concomitant ring opening of either the azetidinyl unit (giving rise to alternative structure 1 for M12 as shown in Fig. 3) or to the oxetanyl ring (alternative structure 2 for M12 as shown in Fig. 3). Similar MS/MS fragment ions exhibiting the loss of the spiro system were also found in M9, M10, and M14.
Formation of the GSH Conjugate (M12) of AZD1979 in Human Liver Subcellular Fractions and with GSTs. To probe the potential involvement of cytochrome P450s in the formation of M12, AZD1979 was incubated in GSH-supplemented human liver subcellular fractions in the presence and absence of $\mathrm{NAD}(\mathrm{P}) \mathrm{H}$. As shown in Fig. 4, M12 was detected in all incubations with human liver cytosol and S9 but not in incubations with mitochondria or microsomes. This result suggested that the enzyme(s) mediating the GSH conjugation of AZD1979 was cytosolic and was operating without prior bioactivation by P450s. In the presence of ethacrynic acid $(100 \mu \mathrm{M})$, a GST inhibitor (Ploemen et al., 1990), the formation of M12 was reduced to $44 \%$ compared with that in control samples in human liver S9 fractions (Fig. 4, inset). Furthermore, the formation of M12 was $\mathrm{pH}$-dependent in human liver S9 fraction incubations, with similar activity at $\mathrm{pH} 6.5$ and 7.4 that was higher in comparison with that at $\mathrm{pH} 8.5$ and 9 (ca. $80 \%-70 \%$ relative to that at $\mathrm{pH} 6.5$ and 7.4).

As shown in Fig. 5, M12 was detected in all incubations of AZD1979 with GSTs. GSTA2-2 exhibited the highest catalytic activity toward M12 formation, followed by GSTM1a and GSTM2-2. Low levels of M12 were also detected in incubations with GSTA1 and GSTT1-1. GST-PLA was reported as containing GSTPi as the major GST isoenzyme (Zusterzeel et al., 1999) and showed the least catalytic activity of M12 formation.

Examination of M12 Formation in Enzyme-Free Buffer Solutions. In view of the potential chemical reactivity of the strained ring systems present in AZD1979, AZD1979 was incubated with GSH, $\mathrm{N}$-acetylcysteine, and/or cysteine in enzyme-free phosphate buffer at $\mathrm{pH}$ $6.5,7.4$, and 9.3 at $37^{\circ} \mathrm{C}$ and $60^{\circ} \mathrm{C}$ for up to 3 hours. None of the corresponding adducts that were detected in enzyme-containing incubations of AZD1979 were formed in aqueous buffer at $37^{\circ} \mathrm{C}$. At $60^{\circ} \mathrm{C}$, the extracted ion chromatogram corresponding to M12 (mass tolerance $20 \mathrm{mDa}$ ) indicated trace amounts of an analyte at the expected retention time after 3 hours at $\mathrm{pH} 7.4$ and 9.3, but the identity of M12 could not be confirmed by MS and MS/MS spectra due to the weakness of the signal corresponding to the mass of M12.

Characterization of the Glutathione Conjugate M12 by NMR. The structure elucidation of the glutathione conjugate M12 by NMR required the isolation of a relatively large amount of pure metabolite. M12 was therefore biosynthesized and purified from scale-up incubations of AZD1979 with rat liver S9 preparations for NMR analysis. The ${ }^{1} \mathrm{H}$ and ${ }^{13} \mathrm{C}$ chemical signals of M12 in acetonitrile- $\mathrm{D}_{3}: \mathrm{D}_{2} \mathrm{O}(1: 1)$ were assigned via their chemical shift and two-dimensional correlations and by comparison to the chemical shifts obtained for AZD1979 in methanol-D 4 . The chemical shifts of AZD1979 and its isolated GSH conjugate M12 are summarized in Table 1. In AZD1979, the two oxetanyl $\mathrm{CH}_{2}(2,4)$ signals in the spiro moiety appear at $\delta 4.73 / 82.2 \mathrm{ppm}$ $\left({ }^{1} \mathrm{H} /{ }^{13} \mathrm{C}\right)$, and the azetidinyl $\mathrm{CH}_{2}(5,7)$ signals at $\delta 3.42 / 63.9 \mathrm{ppm}$. Both groups are singlets integrating as four protons; their carbon signals integrate as two carbons. They both have long-range correlations to the quaternary spiro carbon, $\mathrm{C}(3)$, appearing at $\delta 40.2 \mathrm{ppm}$. The azetidinyl $\mathrm{CH}_{2}$ signals show a long-range correlation to $\mathrm{CH}_{2}(8)$ at $\delta 3.54 / 63.1$ ppm. In the ${ }^{1} \mathrm{H}$ spectrum of M12, the 10 extra nonexchangeable signals from the glutathione residue are readily observed, i.e., protons from four $\mathrm{CH}_{2}$ and two $\mathrm{CH}$ groups. The $\mathrm{CH}_{2}$ (36) connected to sulfur is observed at $\delta 3.26,3.40 / 34.7 \mathrm{ppm}$. A quaternary carbon signal found at $\delta 41.9$ ppm assigned to $\mathrm{C}(3)$ showed long-range correlations to three sets of signals. The first is to a $\mathrm{CH}_{2}$ signal found at $\delta 4.87,4.73 / 78.5 \mathrm{ppm}$, indicating attachment to an oxygen. The ${ }^{1} \mathrm{H}$ signals are split into two doublets, partly obscured by the large water signal; the ${ }^{13} \mathrm{C}$ singlet integrates as two carbons. This signal is assigned as $\mathrm{CH}_{2}$ 's $(4,2)$. The second is to a broadened singlet at $\delta 3.63 / 50.8 \mathrm{ppm}$, a carbon shift indicating attachment to a nitrogen and assigned as $\mathrm{CH}_{2}(7)$ based on 

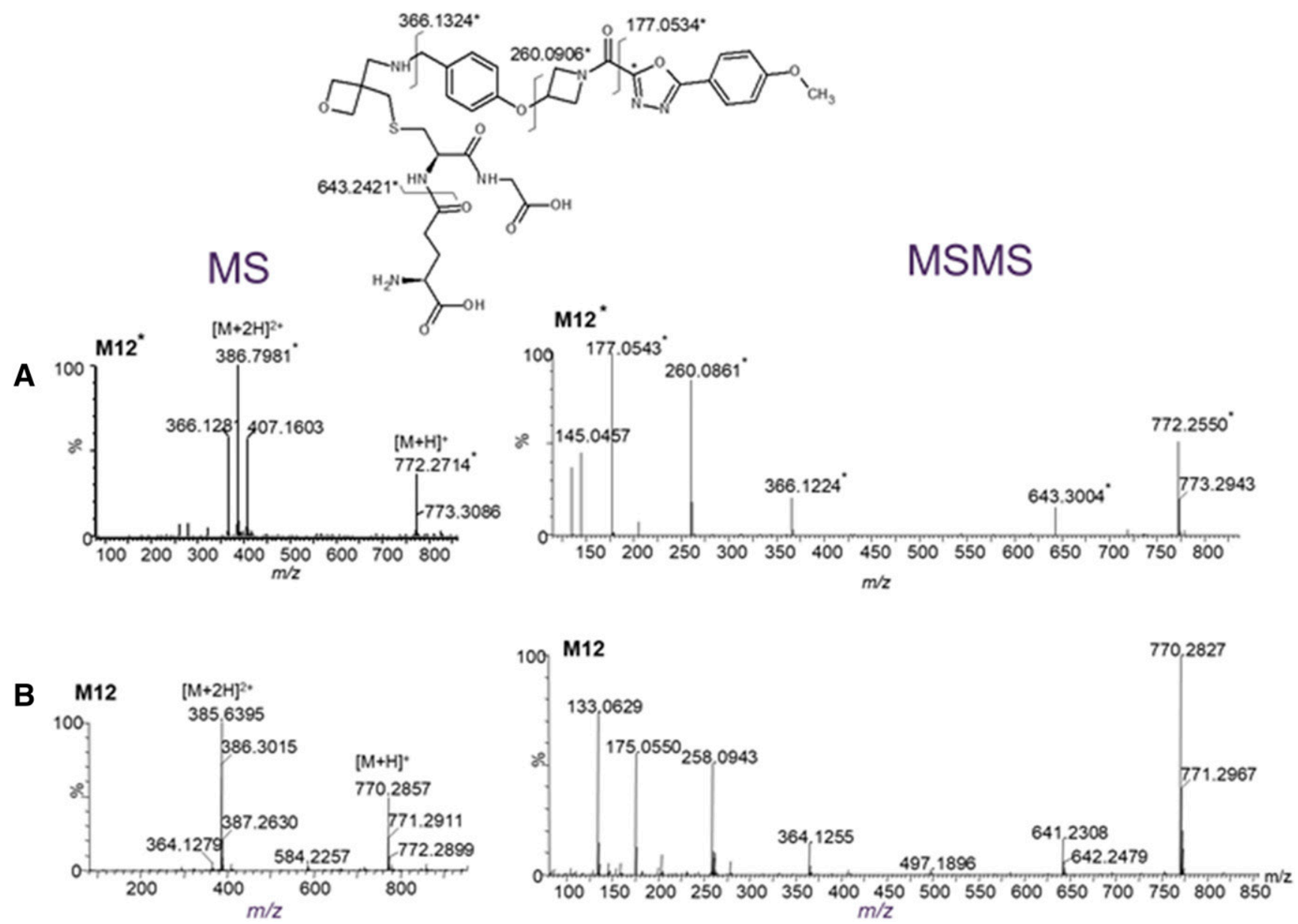

Fig. 2. MS (left panel) and MS/MS (right panel) spectra of GSH-conjugated metabolite M12 formed in the incubation of $\left[{ }^{14} \mathrm{C}\right] \mathrm{AZD} 1979$ in human hepatocytes (A) and the incubation of unlabeled AZD1979 in human liver S9 fraction (B). Asterisks indicate a ${ }^{14} \mathrm{C}$-containing species.

C,H-(long-range)-HMBC correlations to $\mathrm{CH}_{2}$ 's $(2,4)$ and to a $\mathrm{CH}_{2}$ group at 4.51/51.8 identified as $\mathrm{CH}_{2}(8)$. Finally, the quaternary carbon $\mathrm{C}(3)$ at $\delta 41.9 \mathrm{ppm}$ shows a correlation to a sharp singlet, $\mathrm{CH}_{2}(5)$ at $3.38 / 36.4$ ppm, which also correlates to a $\mathrm{CH}_{2}$ group at $\delta 3.26,3.40 / 34.7 \mathrm{ppm}$ identified as $\mathrm{CH}_{2}$ (36) of the GSH moiety (Fig. 6). H,H-ROESY correlations (data not shown) support the data obtained in the $\mathrm{C}, \mathrm{H}-($ longrange)-HMBC spectrum. Due to overlapping signals, ROESY cross peaks between $\mathrm{CH}_{2}$ (5) and $\mathrm{CH}_{2}$ (36) cannot be detected, but ROESY correlations are observed between $\mathrm{CH}_{2}$ (5) and $\mathrm{CH}$ (37) in the glutathione moiety. NMR data unambiguously show that the glutathione is attached to one of the $\mathrm{CH}_{2}$ groups in the spiro-azetidinyl ring.

\section{Discussion}

Metabolite identification plays an important role in drug discovery and development, facilitating the optimization of metabolic stability, the prediction of drug pharmacokinetic/pharmacodynamic properties, and the assessment of potential safety liabilities. LC/HRMS is widely used for metabolite identification because of its excellent selectivity, sensitivity, and the detailed structural information obtained from accurate mass and MS/MS product ion data. However, in some cases, such data may not be sufficient to resolve regiochemical issues. In this study, metabolite M12 exhibited an increase in exact mass of 307.0706 Da compared with parent AZD1979. Such a mass shift is consistent with the net addition of GSH to AZD1979. However, the mass spectral data did not provide insight into the site of attachment of the glutathione. Specifically, it was unclear if the addition of GSH occurred on the spiro-azetidinyl or the oxetanyl ring of the parent molecule. Therefore, the structural characterization of M12 by NMR was pursued. With access to sufficient amounts of biosynthesized M12, NMR data showed unambiguously that GSH bonded via a thiol linkage to the ringopened spiro-azetidinyl moiety of AZD1979, and that the oxetanyl ring remained intact. Thus, alternative structure 1 of M12 in Fig. 3 was shown to be the correct one. On the basis of the identified structure of M12 and the known degradation of GSH adducts via the mercapturic acid pathway (Hinchman and Ballatori, 1990, 1994), the structures of the consecutive metabolites formed from subsequent hydrolysis and modification of the glutathionyl moiety, cysteinylglycinyl (M9), cysteinyl (M10), and the mercapturic acid-conjugated (M14) metabolites, were all assigned with the conjugating site located at the spiroazetidine. The further degradation of the GSH conjugates is mediated by $\gamma$-glutamyl transferase and dipeptidases, i.e., ectoproteins located on the outer surface of, e.g., hepatocytes (Hinchman and Ballatori, 1990, 1994). In this context, it is interesting to note that GSH-conjugated M12 was extensively metabolized via the mercapturic acid pathway to M9, M10, and M14 in our hepatocyte incubations, possibly indicating the efficient transport of intracellularly formed M12 across hepatocyte canalicular or basolateral membranes into the culture medium, thereby accessing $\gamma$-glutamyl transferase and dipeptidases for further degradation.

In this study, the metabolite profiles of $\left[{ }^{14} \mathrm{C}\right] \mathrm{AZD} 1979$ in human hepatocytes revealed that glutathione conjugation was one of the major metabolic pathways of AZD1979. Subsequent investigations were 


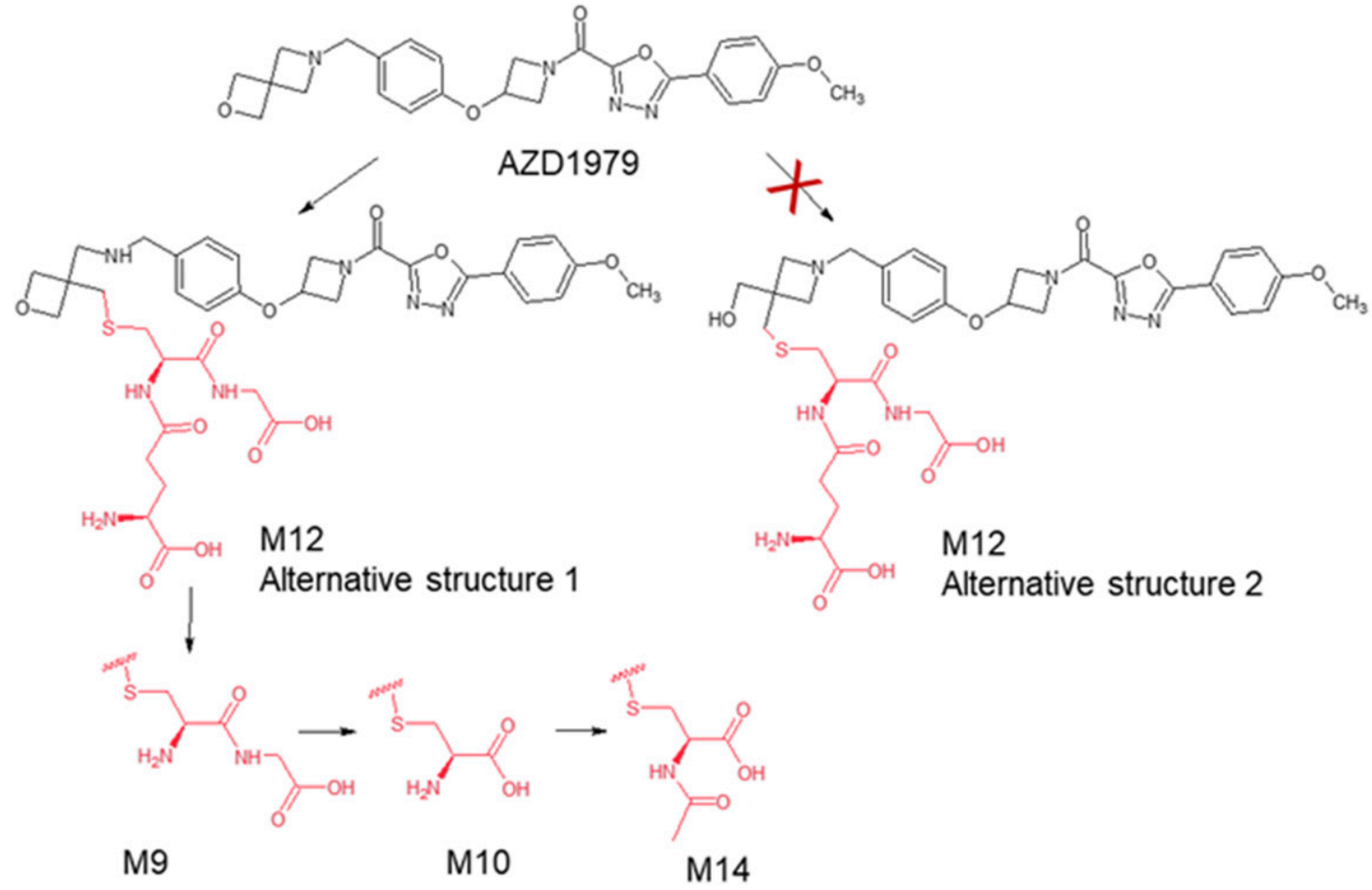

Fig. 3. Proposed metabolic pathways of GSH conjugation of AZD1979 in human hepatocytes.

performed in various human liver preparations in an attempt to understand the biosynthetic pathway leading to this compound. The role of GSTs in the formation of M12 was also investigated. The results of these efforts established the following: 1) the formation of the glutathione conjugate-related metabolites was not inhibited by the

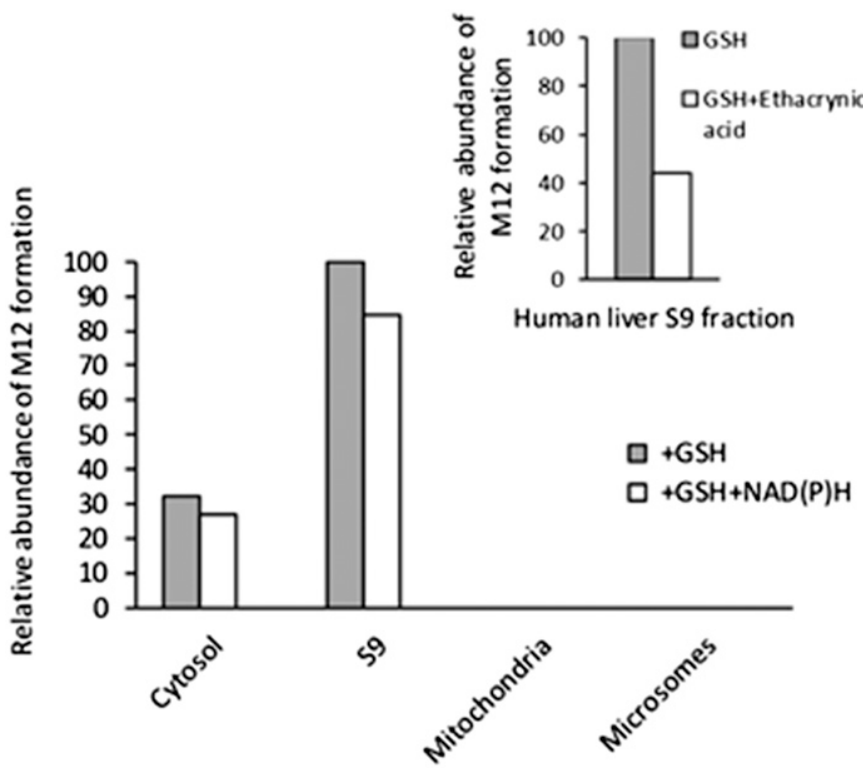

Fig. 4. The effect of $\mathrm{NAD}(\mathrm{P}) \mathrm{H}$ on the formation of $\mathrm{M} 12$ in incubations of human liver subcellular fractions. AZD1979 $(10 \mu \mathrm{M})$ was incubated with various GSHsupplemented human liver subcellular fractions $(1 \mathrm{mg} / \mathrm{ml}$ protein), with and without the presence of a mixture of NADPH and NADH (1 mM each) over 60 minutes. Inset shows the inhibitory effect of ethacrynic acid $(100 \mu \mathrm{M})$ on the formation of M12 in human liver S9 fraction. Results are averages of duplicate measurements. Means of M12 formation in liver S9 incubations without NAD(P) $\mathrm{H}$ were set to $100 \%$. nonselective P450 inhibitor 1-ABT in hepatocytes; 2) the intact GSH conjugate M12 was formed in GSH-supplemented liver cytosol and S9 fractions in an $\mathrm{NAD}(\mathrm{P}) \mathrm{H}$-independent manner but not in microsomal and mitochondrial fractions; 3) M12 formation was inhibited by the GST inhibitor, ethacrynic acid, in liver S9 fractions; and 4) M12 was detected in the incubations of AZD1979 in the presence of isolated GSTs, with GSTA2-2 showing the highest catalytic activity. All of these results together demonstrate that the GSH conjugation pathway of AZD1979 was mediated by cytosolic GSTs without prior bioactivation catalyzed by P450s. In the absence of any enzymes, no M12 formation was detected at $37^{\circ} \mathrm{C}$ and physiologic $\mathrm{pH}$ in aqueous buffers, suggesting the contribution of a spontaneous chemical reaction of AZD1979 with GSH is insignificant. These results also suggest the spiro-azetidinyl moiety of AZD1979 may not react spontaneously with biomacromolecular nucleophiles in vivo. The formation of M12 in human liver S9 fractions was higher than that in cytosol, which may be the result of the different

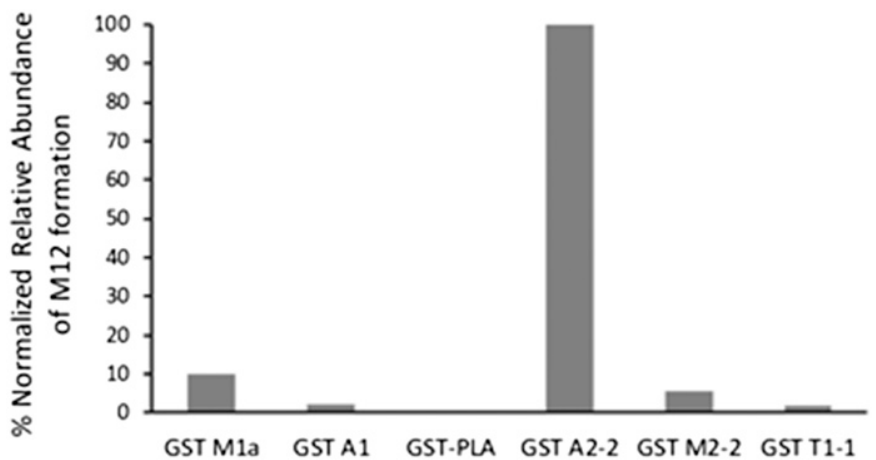

Fig. 5. Formation of M12 in human GSTs. AZD1979 $(10 \mu \mathrm{M})$ was incubated with human GSTs in the presence of GSH $(2 \mathrm{mM})$ over 60 minutes. Formation of M12 was normalized to total protein concentration (MS peak area/mg protein) in the incubations for comparison. Results are averages of duplicate measurements. 
TABLE 1

Proton $\left({ }^{1} \mathrm{H}\right.$ NMR) and carbon $\left({ }^{13} \mathrm{C}\right.$ NMR) chemical shifts of M12 and AZD1979

The numbering system is for illustrative purposes only. NMR data for $\mathrm{M} 12$ acquired at $600-\mathrm{MHz}{ }^{1} \mathrm{H}$ frequency in an approximate $50: 50$ mixture of acetonitrile- $\mathrm{D}_{3}$ and $\mathrm{D}_{2} \mathrm{O}$ at $25^{\circ} \mathrm{C}$ and chemical

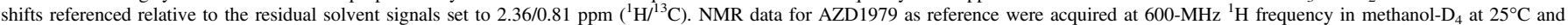
chemical shifts referenced relative to residual solvent signals at $3.33 / 49.0 \mathrm{ppm}\left({ }^{1} \mathrm{H} /{ }^{13} \mathrm{C}\right)$.

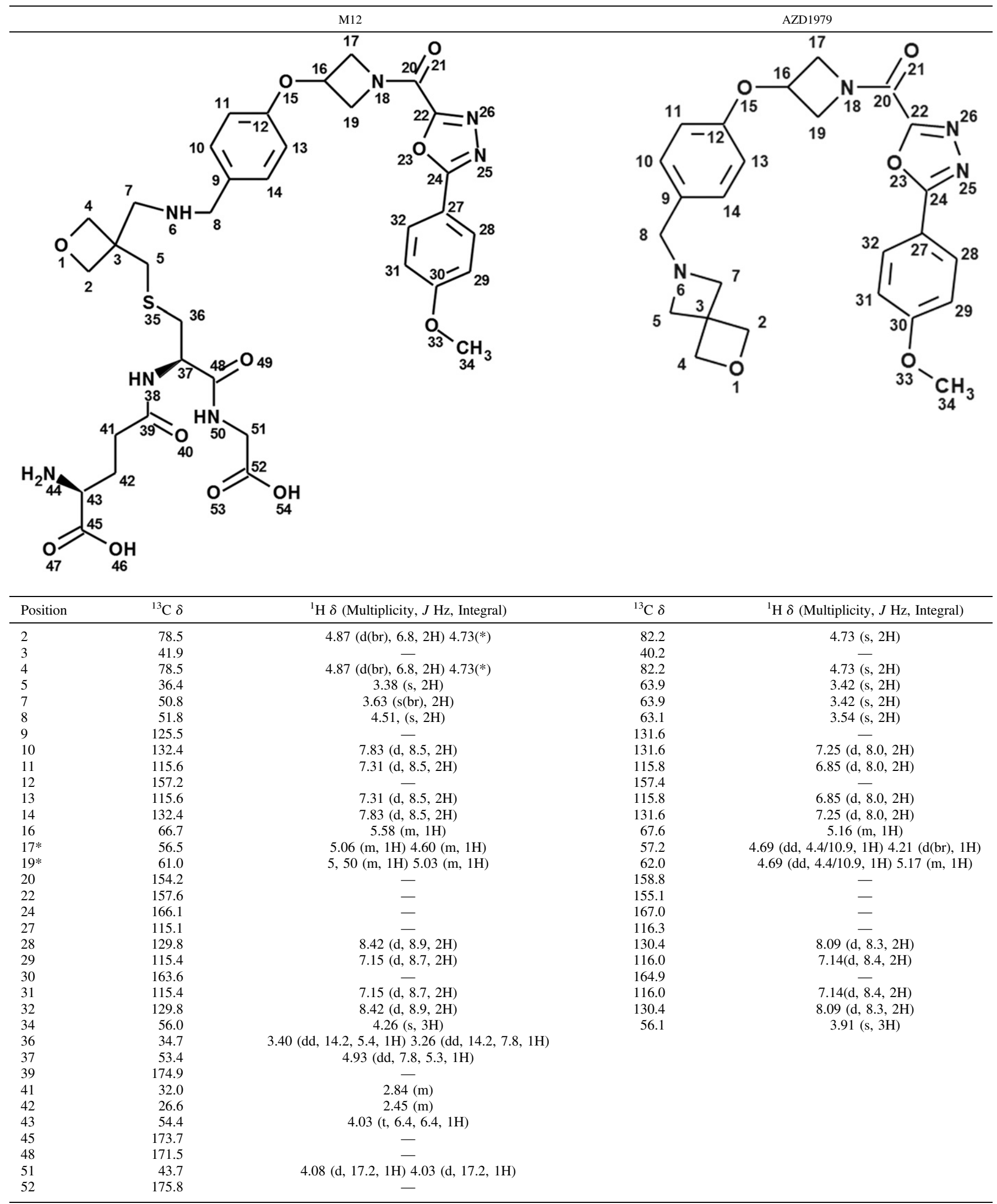




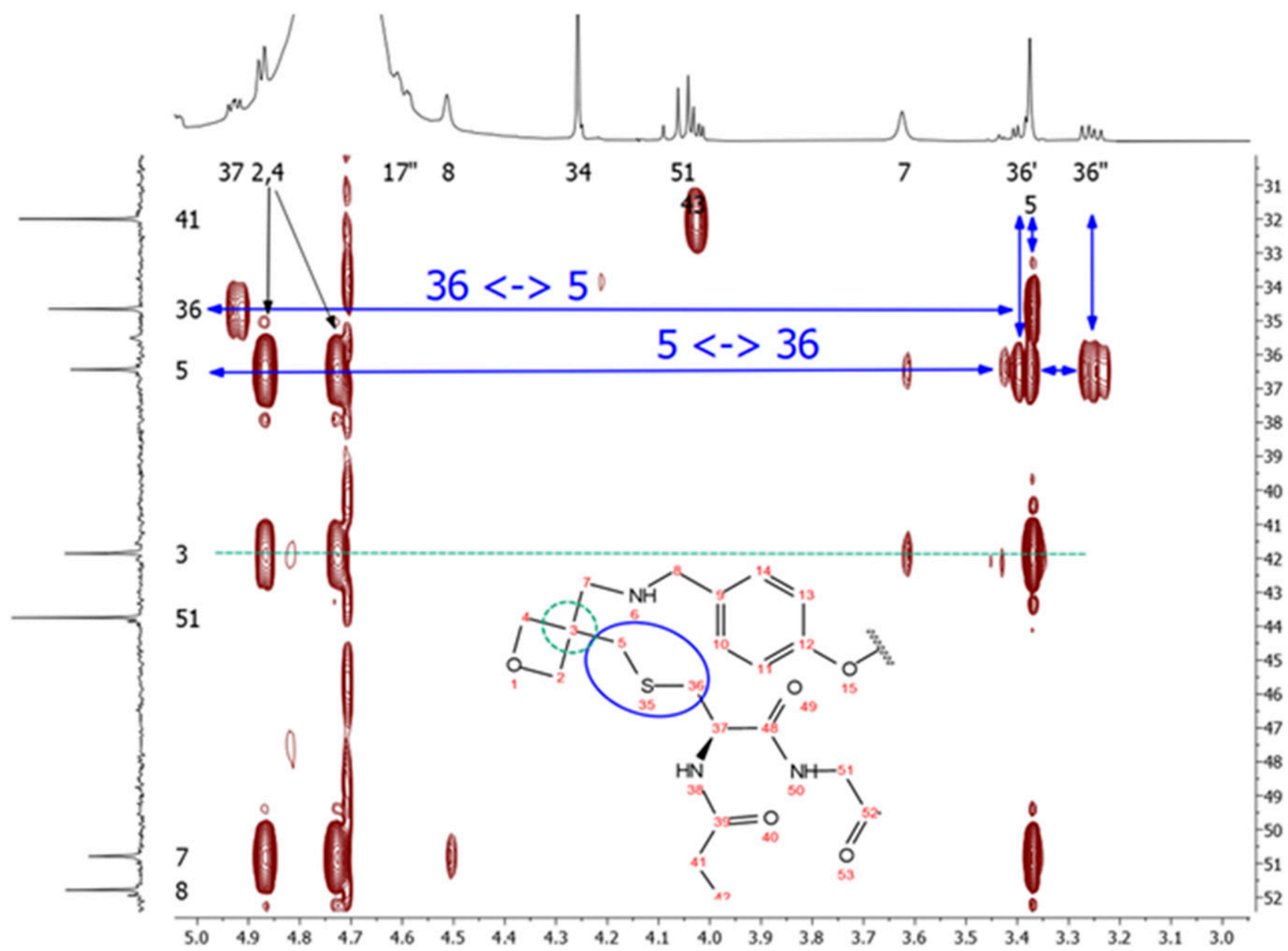

Fig. 6. Diagnostic ${ }^{1} \mathrm{H}_{-}{ }^{13} \mathrm{C}$ long-rang correlations of the isolated metabolite $\mathrm{M} 12$ observed in two-dimensional $\mathrm{HMBC}$ NMR spectra. $\mathrm{Cross}$ peaks correlating $\mathrm{CH}_{2}(36)$ of the glutathione moiety to $\mathrm{CH}_{2}(5)$ of M12.

sources of these two liver subcellular preparations. Due to this observation, the liver S9 fraction was further used to investigate the impact of $\mathrm{pH}$ and the presence of the GST inhibitor ethacrynic acid on M12 formation.

Glutathione $S$-transferases, including cytosolic, mitochondrial, and microsomal GSTs, are from a family of enzymes that catalyze the conjugation of a wide variety of electrophilic substrates to GSH (Hayes et al., 2005; Oakley, 2011; Wu and Dong, 2012). Human cytosolic GSTs are one of the major conjugating enzyme systems responsible for the metabolism of xenobiotics (Wu and Dong, 2012; Mohana and Achary, 2017), and they have been assigned into seven classes: GST $\alpha, \mu, \pi, \sigma$, $\theta, \omega$, and $\zeta$ (Mannervik et al., 2005). Substrates for GSTs are electrophilic compounds and include metabolic intermediates such as epoxides, arene oxides, quinones, quinoneimines, sulfoxides, cation radicals, and substrates susceptible to conjugating addition reactions, such as those containing $\alpha, \beta$-unsaturated ketones (van Bladeren, 2000; Jancova et al., 2010). GSTs catalyze the attack of the nucleophilic thiolate anion of GSH $\left(\mathrm{GS}^{-}\right)$on these electrophilic centers to yield conjugate addition products. Many of these electrophilic species also can react spontaneously with endogenous GSH, although GSTs clearly play an important role in the detoxification of many electrophilic substrates. More recently, drugs have been designed to target nucleophiles, e.g., to covalently modify tumor-specific targets via Michael addition, where GSH adducts were detected as nonenzyme-mediated metabolites (Shibata and Chiba, 2015). Reports of xenobiotics that undergo GSH conjugation requiring catalysis by GSTs but that do not need prior metabolic activation are few, e.g., Yuan et al. (1991) reported that phosphoramide mustard undergoes GSH conjugation in a reaction catalyzed by hepatic GSTs. They proposed that the enzyme-catalyzed conjugation took place via an intermediate cyclic aziridinium ion followed by ring-opening resulting from the addition of GSH. Our study showed that the formation of M12 was also catalyzed by GSTs, and the nucleophilic reaction of AZD1979 by GSH was on its highenergy, ring-strained spiro-azetidinyl unit. We hereby propose a mechanism shown in Fig. 7 to account for the formation of M12. This pathway is analogous to that presented by Yuan et al. (1991) since they both proceed via a protonated strained cyclic aminyl intermediate that should be highly susceptible to attack by the $\mathrm{GS}^{-}$species in a GST-catalyzed reaction. The ability to form such protonated intermediate is clearly very important for further GSH conjugation and ring opening. GSTs catalyze the conjugation of GSH to a xenobiotic through lowering the $\mathrm{pK}_{\mathrm{a}}$ of $\mathrm{GSH}$ from 9 to 6.4-6.7 and stabilizing the thiolate anion through a hydrogen bonding (Armstrong, 1991). It is not difficult to anticipate that a proton source strategically located in the GST active site or derived from GSH could facilitate the nucleophilic attack of $\mathrm{GS}^{-}$by providing azetidiniumyl character to the substrate. The optimal $\mathrm{pH}$ of 6.5-7.4 for M12 formation in liver S9 fraction satisfied both the formation of the $\mathrm{GS}^{-}$species via the GST-bound GSH $\left(\mathrm{pK}_{\mathrm{a}} 6.4-6.7\right)$ and the formation of protonated azetidiniumyl species $\left(\mathrm{pK}_{\mathrm{a}}\right.$ at 8.5$)$. The lack of chemical reactivity of the azetidinyl moiety with GSH in aqueous 

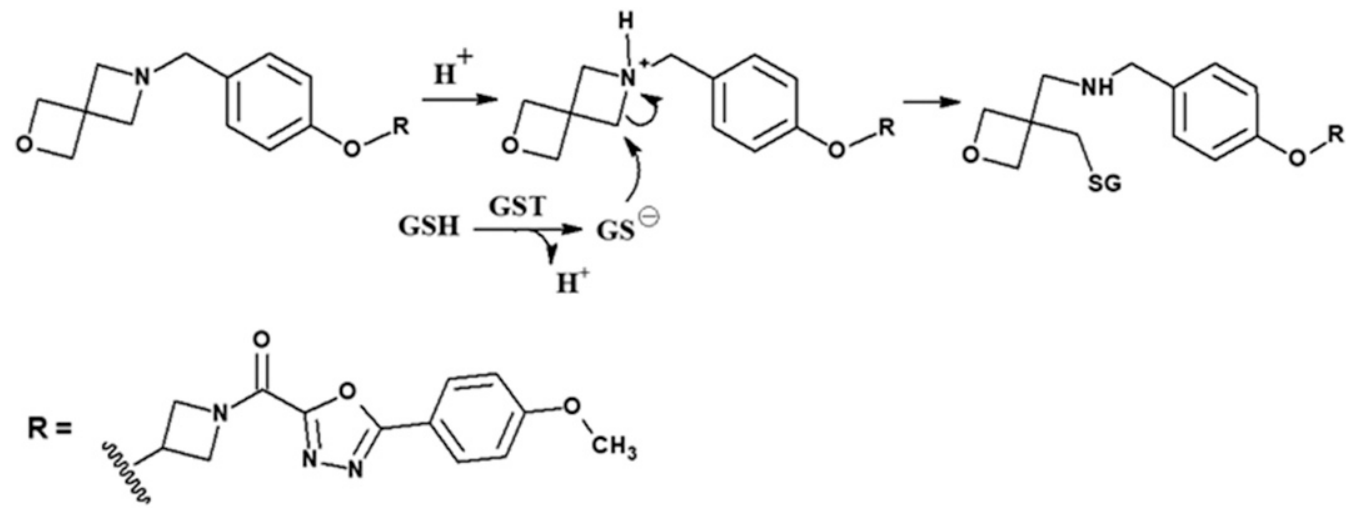

Fig. 7. Proposed mechanism of glutathione addition to the spiro-azetidine ring of AZD1979.

solution can also be explained by the absence of either the protonated azetidiniumyl or the $\mathrm{GS}^{-}$species. Our previous studies (Li et al., 2016; Toselli et al., 2017) revealed that the oxetanyl rings in the spirooxetanylazetidinyl moiety in AZD1979 and its oxetane-containing analogs also undergo an unusual non-P450-catalyzed reaction with the formation of oxetanyl ring-opened diol mediated by microsomal epoxide hydrolase. All of these findings highlighted the potential reactivity of high-energy, ring-strained-containing xenobiotics that may be subject to unexpected metabolic reactions. However, an important conclusion is also that the ring strain of the azetidine is not sufficient to promote spontaneous reactions with nucleophiles, e.g., hydrolysis or GSH adduction. The analogous situation has been observed for the oxetanyl moiety that undergoes ring-opening hydration only in the presence of microsomal epoxide hydrolase (Li et al., 2016). Thus, although the GSH conjugate was indeed formed, this finding does not suggest a general reactivity of this spiro moiety toward biomacromolecules. Further studies will be required to gain insight into the structural features of azetidinyl-containing compounds that render them susceptible to GSH conjugation. With the knowledge that GSTs catalyze this reaction, a well defined structure-activity relationship study will be undertaken to further our knowledge of the extent to which high-energy, ring-strained amines may be susceptible to GSH conjugation.

The importance of non-P450-mediated metabolism in drug discovery has been the focus of numerous studies during recent years (Barski et al., 2008; Wu et al., 2011; Gan et al., 2016; Hollenberg, 2016; Marto et al., 2017; Shi and Di, 2017; Toselli et al., 2017). Drugs cleared in humans principally by non-P450-mediated pathways have led to poor predictions of exposure and/or toxicity (Diamond et al., 2010; Akabane et al., 2011; Meng et al., 2015; Zhuo et al., 2016). GST enzymes are extensively distributed in human tissues, and in general, GSH conjugation is regarded as a detoxification reaction leading to the formation of chemically less-reactive products. We have established GSH conjugation to be a significant metabolic pathway of AZD1979 in human hepatocytes, and given that human cytosolic GST isozymes exhibit genetic polymorphisms and show large variabilities in expression levels between individuals (Hayes et al., 2005; den Braver et al., 2016), further investigations of the contributions of specific GST isoforms to AZD1979 metabolism would be valuable to understand their roles in AZD1979 metabolism and disposition in vivo.

In summary, we have discovered an unusual GSH conjugation of an azetidinyl ring in a spiro-oxetanylazetidinyl-containing drug candidate, which is catalyzed by GSTs with no requirement for prior bioactivation by $\mathrm{P} 450 \mathrm{~s}$. It demonstrates the inherent chemical reactivities of highenergy, ring-strained functional groups and shows that the chemical reactivity may be enhanced by the biologic environment, i.e., the GSH conjugation reaction of AZD1979 is mediated by GSTs. The chemical reactivity and metabolic fate of a compound lead to consideration of the possible link between chemical reactivity and biologic (toxicological) properties of compounds. In our study, the GST-mediated metabolic pathway of AZD1979 was also apparent in rat liver S9 fractions, supporting the coverage of this pathway in an animal species relevant to the safety assessment of metabolites likely to be found also in clinical studies. The finding that GSH-conjugated metabolites of AZD1979 were formed was not regarded as a major toxicological concern, and the biotransformation profiles across species were considered acceptable for the advancement of AZD1979 into clinical development.

\section{Acknowledgments}

The authors thank Drs. Moa Andersson-Bergström, Britta Bonn, and Lars Dalmin for conducting part of the in vitro experiments.

\section{Authorship Contributions}

Participated in research design: Li, Hayes, Castagnoli, Weidolf.

Conducted experiments: Li, Grönberg, Bangur.

Performed data analysis: Li, Grönberg, Bangur.

Wrote or contributed to the writing of the manuscript: Li, Hayes, Grönberg, Castagnoli, Weidolf.

\section{References}

Akabane T, Tanaka K, Irie M, Terashita S, and Teramura T (2011) Case report of extensive metabolism by aldehyde oxidase in humans: pharmacokinetics and metabolite profile of FK3453 in rats, dogs, and humans. Xenobiotica 41:372-384.

Armstrong RN (1991) Glutathione S-transferases: reaction mechanism, structure, and function. Chem Res Toxicol 4:131-140.

Barski OA, Tipparaju SM, and Bhatnagar A (2008) The aldo-keto reductase superfamily and its role in drug metabolism and detoxification. Drug Metab Rev 40:553-624.

Dekker SJ, Zhang Y, Vos JC, Vermeulen NPE, and Commandeur JNM (2016) Different reactive metabolites of nevirapine require distinct glutathione $\mathrm{S}$-transferase isoforms for bioinactivation. Chem Res Toxicol 29:2136-2144.

den Braver MW, Zhang Y, Venkataraman H, Vermeulen NPE, and Commandeur JNM (2016) Simulation of interindividual differences in inactivation of reactive para-benzoquinone imine metabolites of diclofenac by glutathione S-transferases in human liver cytosol. Toxicol Lett $\mathbf{2 5 5}$ : $52-62$.

Diamond S, Boer J, Maduskuie TP Jr, Falahatpisheh N, Li Y, and Yeleswaram S (2010) Speciesspecific metabolism of SGX523 by aldehyde oxidase and the toxicological implications. Drug Metab Dispos 38:1277-1285.

Dragovic S, Boerma JS, van Bergen L, Vermeulen NP, and Commandeur JN (2010) Role of human glutathione S-transferases in the inactivation of reactive metabolites of clozapine. Chem Res Toxicol 23:1467-1476.

Emoto C, Murase S, Sawada Y, Jones BC, and Iwasaki K (2003) In vitro inhibitory effect of 1aminobenzotriazole on drug oxidations catalyzed by human cytochrome P450 enzymes: a comparison with SKF-525A and ketoconazole. Drug Metab Pharmacokinet 18:287-295.

Gan J, Ma S, and Zhang D (2016) Non-cytochrome P450-mediated bioactivation and its toxicological relevance. Drug Metab Rev 48:473-501.

Hayes JD, Flanagan JU, and Jowsey IR (2005) Glutathione transferases. Аnпи Rev Pharmacol Toxicol 45:51-88.

Hinchman CA and Ballatori N (1990) Glutathione-degrading capacities of liver and kidney in different species. Biochem Pharmacol 40:1131-1135.

Hinchman CA and Ballatori N (1994) Glutathione conjugation and conversion to mercapturic acids can occur as an intrahepatic process. J Toxicol Environ Health 41:387-409.

Hollenberg PF (2016) Enzymology in chemical toxicology: beyond P450s. Chem Res Toxicol 29: $1365-1366$. 
Jancova P, Anzenbacher P, and Anzenbacherova E (2010) Phase II drug metabolizing enzymes. Biomed Pap Med Fac Univ Palacky Olomouc Czech Repub 154:103-116.

Johansson A, Löfberg C, Antonsson M, von Unge S, Hayes MA, Judkins R, Ploj K, Benthem L, Lindén D, Brodin P, et al. (2016) Discovery of (3-(4-(2-oxa-6-azaspiro[3.3]heptan-6-ylmethyl) phenoxy)azetidin-1-yl)(5-(4-methoxyphenyl)-1,3,4-oxadiazol-2-yl)methanone (AZD1979), a melanin concentrating hormone receptor $1(\mathrm{MCHr} 1)$ antagonist with favorable physicochemical properties. J Med Chem 59:2497-2511.

Li XQ, Hayes MA, Grönberg G, Berggren K, Castagnoli N Jr, and Weidolf L (2016) Discovery of a novel microsomal epoxide hydrolase-catalyzed hydration of a spiro oxetane. Drug Metab Dispos 44:1341-1348.

Mannervik B, Board PG, Hayes JD, Listowsky I, and Pearson WR (2005) Nomenclature for mammalian soluble glutathione transferases. Methods Enzymol 401:1-8.

Marto N, Morello J, Monteiro EC, and Pereira SA (2017) Implications of sulfotransferase activity in interindividual variability in drug response: clinical perspective on current knowledge. Drug Metab Rev 49:357-371.

Meng J, Zhong D, Li L, Yuan Z, Yuan H, Xie C, Zhou J, Li C, Gordeev MF, Liu J, et al. (2015) Metabolism of MRX-I, a novel antibacterial oxazolidinone, in humans: the oxidative ring opening of 2,3-Dihydropyridin-4-one catalyzed by non-P450 enzymes. Drug Metab Dispos 43: 646-659.

Mohana K and Achary A (2017) Human cytosolic glutathione-S-transferases: quantitative analysis of expression, comparative analysis of structures and inhibition strategies of isozymes involved in drug resistance. Drug Metab Rev 49:318-337.

Munter T, Cottrell L, Golding BT, and Watson WP (2003) Detoxication pathways involving glutathione and epoxide hydrolase in the in vitro metabolism of chloroprene. Chem Res Toxicol 16:1287-1297.

Oakley A (2011) Glutathione transferases: a structural perspective. Drug Metab Rev 43:138-151.

Okada R, Maeda K, Nishiyama T, Aoyama S, Tozuka Z, Hiratsuka A, Ikeda T, Kusuhara H, and Sugiyama Y (2011) Involvement of different human glutathione transferase isoforms in the glutathione conjugation of reactive metabolites of troglitazone. Drug Metab Dispos 39: 2290-2297.

Ploemen JH, van Ommen B, and van Bladeren PJ (1990) Inhibition of rat and human glutathione S-transferase isoenzymes by ethacrynic acid and its glutathione conjugate. Biochem Pharmacol 40:1631-1635.
Shi SM and Di L (2017) The role of carbonyl reductase 1 in drug discovery and development. Expert Opin Drug Metab Toxicol 13:859-870.

Shibata Y and Chiba M (2015) The role of extrahepatic metabolism in the pharmacokinetics of the targeted covalent inhibitors afatinib, ibrutinib, and neratinib. Drug Metab Dispos 43 375-384.

Toselli F, Fredenwall M, Svensson P, Li XQ, Johansson A, Weidolf L, and Hayes MA (2017) Oxetane substrates of human microsomal epoxide hydrolase. Drug Metab Dispos 45:966-973.

van Bladeren PJ (2000) Glutathione conjugation as a bioactivation reaction. Chem Biol Interact 129:61-76.

Wu B and Dong D (2012) Human cytosolic glutathione transferases: structure, function, and drug discovery. Trends Pharmacol Sci 33:656-668.

Wu B, Kulkarni K, Basu S, Zhang S, and Hu M (2011) First-pass metabolism via UDP-glucuronosyltransferase: a barrier to oral bioavailability of phenolics. J Pharm Sci 100:3655-3681.

Yuan ZM, Smith PB, Brundrett RB, Colvin M, and Fenselau C (1991) Glutathione conjugation with phosphoramide mustard and cyclophosphamide. A mechanistic study using tandem mass spectrometry. Drug Metab Dispos 19:625-629.

Zhuo X, Cantone JL, Wang Y, Leet JE, Drexler DM, Yeung K-S, Huang XS, Eastman KJ, Parcella KE, Mosure KW, et al. (2016) Phosphocholine conjugation: an unexpected in vivo conjugation pathway associated with hepatitis c ns5b inhibitors featuring a bicyclo[1.1.1]pentane. Drug Metab Dispos 44:1332-1340.

Zhuo X, Huang XS, Degnan AP, Snyder LB, Yang F, Huang H, Shu YZ, and Johnson BM (2015) Identification of glutathione conjugates of acetylene-containing positive allosteric modulators of metabotropic glutamate receptor subtype 5. Drug Metab Dispos 43:578-589.

Zusterzeel PL, Peters WH, De Bruyn MA, Knapen MF, Merkus HM, and Steegers EA (1999) Glutathione S-transferase isoenzymes in decidua and placenta of preeclamptic pregnancies. Obstet Gynecol 94:1033-1038.

Address correspondence to: Dr. Lars Weidolf, Research and Early Development, Cardiovascular, Renal and Metabolism, BioPharmaceuticals R\&D, AstraZeneca, Gothenburg, Sweden. E-mail: lars.weidolf@astrazeneca.com 